\title{
Site-specific metabolic phenotypes in metastatic breast cancer
}

\author{
Hye Min Kim, Woo Hee Jung and Ja Seung Koo*
}

\begin{abstract}
Background: The purpose of this study was to examine the expression of metabolism-related proteins according to metastatic site in metastatic breast cancer and to assess the implication of site-specific differential expression.

Methods: A tissue microarray containing 162 cases of metastatic breast cancer (52 lung metastasis, 47 bone metastasis, 39 brain metastasis, and 24 liver metastasis) was constructed. It was subject to immunohistochemical staining of the following proteins: Glycolysis-related: Glut-1, hexolinase II, carbonic anhydrase (CA) IX, and monocarboxylate transporter (MCT) 4; glutaminolysis-related: glutaminase (GLS) 1, glutamate dehydrogenase (GDH), and amino acid transporter (ASCT) 2; mitochondrial metabolism-related: ATP synthase, succinate dehydrogenase (SDH)A, and SDHB; and serine/glycine metabolism related: phosphoglycerate dehydrogenase (PHGDH), phosphoserine aminotransferase (PSAT), phosphoserine phosphatase (PSPH), glycine decarboxylase (GLDC), and serine hydroxymethyltransferase (SHMT).
\end{abstract}

Results: The expression levels of glycolysis-related-proteins (Glut-1, hexokinase II, CAIX, and MCT4) differed according to metastatic site, with higher expression seen in the brain and lower expression in the bone and liver $(p<0.001,0.001$, 0.009 , and $<0.001$, respectively). Differences in metabolic phenotype were analyzed according to metastasis site. Glycolysis type was most frequently encountered in the brain and lung $(p<0.001)$. In univariate analysis, the factors associated with shorter overall survival were CAIX positivity $(p=0.044)$, PSPH positivity $(p=0.045)$, and SHMT1 positivity $(p=0.002)$, as well as serine/glycine type $(p=0.041)$.

Conclusions: Differences in metabolic features according to metastatic site were seen in metastatic breast cancer, with the glycolysis phenotype found predominantly in the brain and lung and the non-glycolysis phenotype in the bone and liver.

Keywords: Breast, Metabolism, Metastasis

\section{Introduction}

Breast cancer has a high morbidity and mortality, mainly because it can easily metastasize to distant organs. The main metastatic sites from breast cancer are the lung, bone, brain, and liver [1,2]. However, most studies have focused on bone and brain metastases [3-8]. The main mechanism of tumor metastasis is the reciprocal interaction between tumor cells and the host tissue, involving cell adhesion, proteolysis, invasion, and angiogenesis $[2,9]$. Because different cancers display distinct metastatic patterns, the seed and soil hypothesis has been proposed, which dictates that the specific tumor (seed) can survive only in specific visceral organs (soil) [10].

\footnotetext{
*Correspondence: kjs1976@yuhs.ac

Department of Pathology, Severance Hospital, Yonsei University College of Medicine, 50 Yonsei-ro, Seodaemun-gu, Seoul 120-752, South Korea
}

Accordingly, metastatic breast cancer cells show different characteristics according to the metastatic site. For example, brain metastasis is associated with young age, estrogen receptor (ER) negativity, prior lung metastasis, HER-2 overexpression, EGFR overexpression, and the basal subtype [5-7], while bone metastasis is associated with lower histologic grade, ER positivity, ER positivity/progesterone receptor (PR) negativity, strand growth pattern, and the presence of fibrotic foci in invasive ductal carcinoma $[4,11,12]$. Therefore, metastatic breast cancer is also likely to display distinct characteristics according to metastatic site.

According to the Warburg effect theory, while normal cells gain energy from oxidative phosphorylation, cancer cells obtain energy from glycolysis, making glycolysis an important component in cancer metabolism [13]. However, this theory cannot fully explain the energy usage of all 
Table 1 Clone, dilution, and source of antibodies used in this study

\begin{tabular}{|c|c|c|c|}
\hline Antibody & Clone & Dilution & Source \\
\hline \multicolumn{4}{|c|}{ Molecular subtype related } \\
\hline ER & SP1 & $1: 100$ & Thermo Scientific, CA, USA \\
\hline$P R$ & $\mathrm{PgR}$ & $1: 50$ & DAKO, Denmark \\
\hline HER-2 & Polyclonal & $1: 1500$ & DAKO, Denmark \\
\hline Ki-67 & MIB-1 & $1: 150$ & DAKO, Denmark \\
\hline \multicolumn{4}{|l|}{ Glycolysis related } \\
\hline Glut-1 & SPM498 & $1: 200$ & Abcam, Cambridge, UK \\
\hline Hexokinase II & 3D3 & $1: 200$ & Abcam, Cambridge, UK \\
\hline CAIX & Polyclonal & $1: 100$ & Abcam, Cambridge, UK \\
\hline MCT4 & Polyclonal & $1: 100$ & Santa Cruz, CA, USA \\
\hline \multicolumn{4}{|c|}{ Glutaminolysis related } \\
\hline GLS1 & Polyclonal & $1: 50$ & Abcam, Cambridge, UK \\
\hline $\mathrm{GDH}$ & Polyclonal & $1: 100$ & Abcam, Cambridge, UK \\
\hline ASCT2 & Polyclonal & $1: 100$ & Abcam, Cambridge, UK \\
\hline \multicolumn{4}{|c|}{ Mitochondrial related } \\
\hline ATP synthase & $15 \mathrm{H} 4 \mathrm{C} 4$ & $1: 100$ & Abcam, Cambridge, UK \\
\hline SDHA & 2E3GC12FB2AE2 & $1: 100$ & Abcam, Cambridge, UK \\
\hline SDHB & 21A11AE7 & $1: 100$ & Abcam, Cambridge, UK \\
\hline \multicolumn{4}{|c|}{ Serine/glycine metabolism related } \\
\hline PHGDH & Polyclonal & $1: 100$ & Abcam, Cambridge, UK \\
\hline PSPH & Polyclonal & $1: 100$ & Abcam, Cambridge, UK \\
\hline PSAT1 & Polyclonal & $1: 100$ & Abcam, Cambridge, UK \\
\hline SHMT & Polyclonal & $1: 100$ & Abcam, Cambridge, UK \\
\hline GLDC & Polyclonal & 1:100 & Abcam, Cambridge, UK \\
\hline
\end{tabular}

Table 2 Basal clinicopathologic characteristics of patients with breast cancer metastasis according to metastatic site

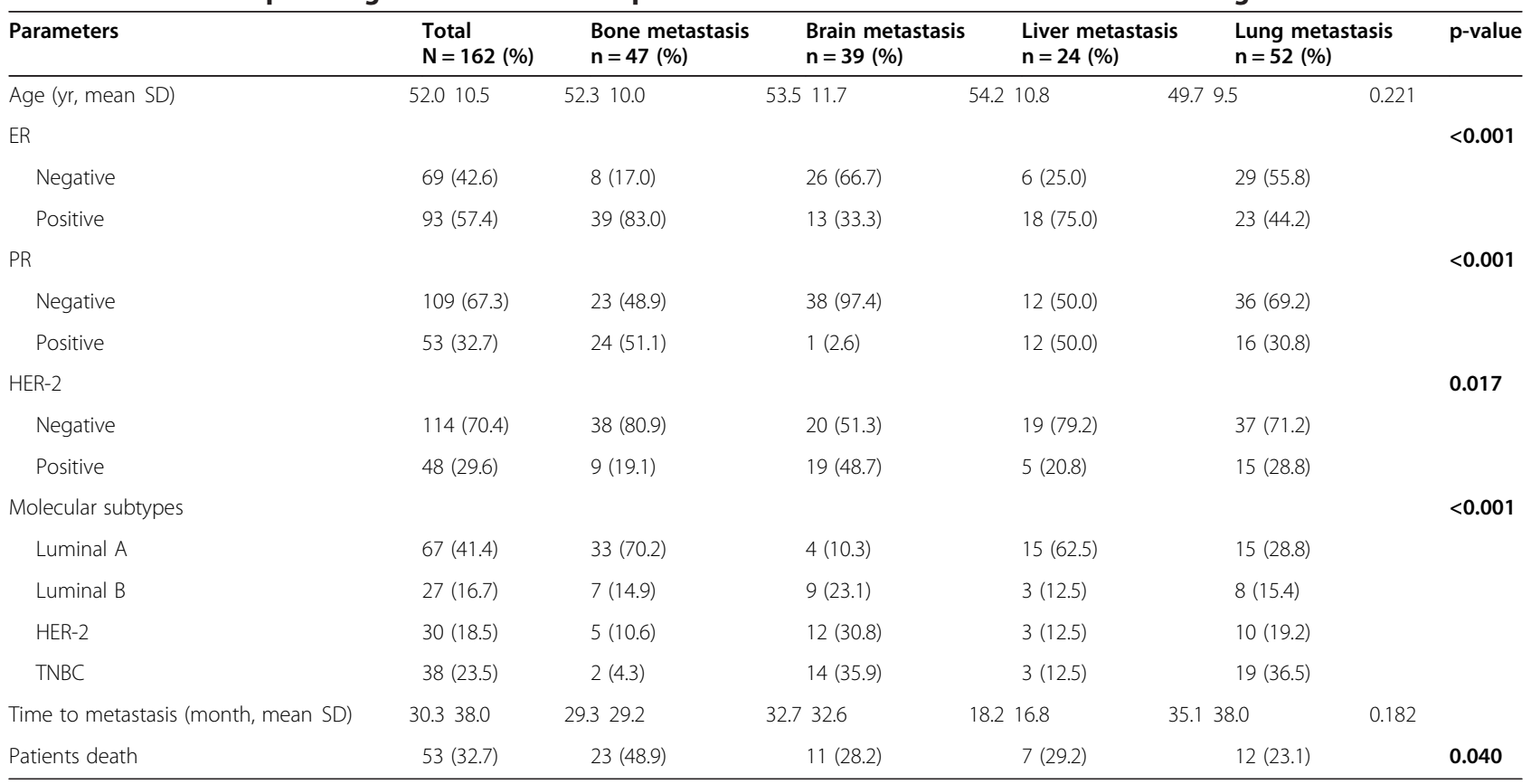

Bold represents $\mathrm{p}<0.05$. 
cancer cells [14]. Glutamine and mitochondrial metabolism, along with glucose metabolism, are also important components in cancer cell metabolism. Tumor cells under active glycolysis have higher levels of glycolytic intermediates, and the metabolism of glycolytic intermediates has been recently shown to be involved in tumorigenesis. A representative metabolic pathway of glycolytic intermediates is the glycine and serine metabolic pathway [15-18], which has been recently studied as a new possible target for tumor therapy [19]. Targeted therapy can be used in metastatic cancer, as well as in primary cancer, making the identification of metabolic phenotypes in metastatic cancer clinically important. However, metastatic cancer displays distinct characteristics according to metastatic site, but the sitespecific metabolic features have not yet been fully identified. The purpose of this study was to examine the expression of metabolism-related proteins according to their metastatic site in metastatic breast cancer and their implication.

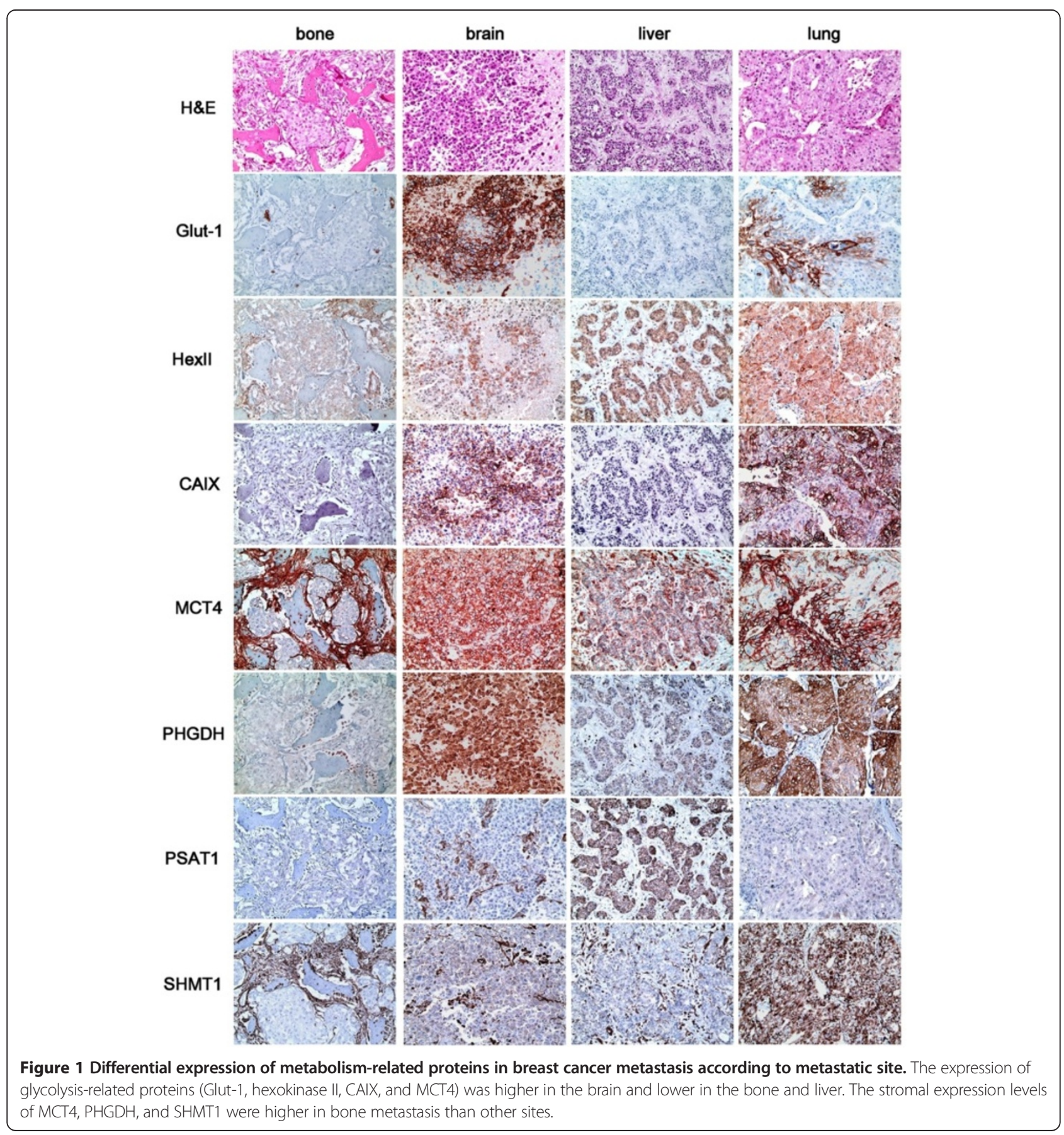


Table 3 Expression of metabolism-related proteins in the tumor cell compartment of breast cancer metastasis according to metastatic site

\begin{tabular}{|c|c|c|c|c|c|c|}
\hline Parameters & $\begin{array}{l}\text { Total } \\
\mathrm{N}=162 \text { (\%) }\end{array}$ & $\begin{array}{l}\text { Bone metastasis } \\
n=47(\%)\end{array}$ & $\begin{array}{l}\text { Brain metastasis } \\
\mathrm{n}=39(\%)\end{array}$ & $\begin{array}{l}\text { Liver metastasis } \\
\mathrm{n}=24(\%)\end{array}$ & $\begin{array}{l}\text { Lung metastasis } \\
\mathrm{n}=52(\%)\end{array}$ & p-value \\
\hline Glut-1 & & & & & & $<0.001$ \\
\hline Negative & $83(51.2)$ & $35(74.5)$ & $10(25.6)$ & $18(75.0)$ & $20(38.5)$ & \\
\hline Positive & 79 (48.8) & $12(25.5)$ & $29(74.4)$ & $6(25.0)$ & $32(61.5)$ & \\
\hline Hexokinase II & & & & & & 0.001 \\
\hline Negative & $113(69.8)$ & $41(87.2)$ & $25(64.1)$ & 20 (83.3) & 27 (51.9) & \\
\hline Positive & $49(30.2)$ & $6(12.8)$ & $14(35.9)$ & $4(16.7)$ & $25(48.1)$ & \\
\hline CAIX & & & & & & 0.009 \\
\hline Negative & $130(80.2)$ & 44 (93.6) & $26(66.7)$ & $21(87.5)$ & $39(75.0)$ & \\
\hline Positive & 32 (19.8) & $3(6.4)$ & 13 (33.3) & $3(12.5)$ & $13(25.0)$ & \\
\hline MCT4 & & & & & & $<0.001$ \\
\hline Negative & $66(40.7)$ & $25(53.2)$ & $4(10.3)$ & $13(54.2)$ & $24(46.2)$ & \\
\hline Positive & $96(59.3)$ & $22(46.8)$ & 35 (89.7) & $11(45.8)$ & $28(53.8)$ & \\
\hline GLS1 & & & & & & 0.473 \\
\hline Negative & $83(51.2)$ & 28 (59.6) & 17 (43.6) & $11(45.8)$ & 27 (51.9) & \\
\hline Positive & 79 (48.8) & 19 (40.4) & $22(56.4)$ & $13(54.2)$ & $25(48.1)$ & \\
\hline $\mathrm{GDH}$ & & & & & & 0.610 \\
\hline Negative & $2(1.2)$ & $1(2.1)$ & $1(2.6)$ & $0(0.0)$ & $0(0.0)$ & \\
\hline Positive & $160(98.8)$ & $46(97.9)$ & 38 (97.4) & $24(100.0)$ & $52(100.0)$ & \\
\hline ASCT2 & & & & & & 0.033 \\
\hline Negative & $122(75.3)$ & $37(78.7)$ & $34(87.2)$ & $19(79.2)$ & $32(61.5)$ & \\
\hline Positive & $40(24.7)$ & $10(21.3)$ & $5(12.8)$ & $5(20.8)$ & $20(38.5)$ & \\
\hline ATP synthase & & & & & & 0.610 \\
\hline Negative & $2(1.2)$ & $1(2.1)$ & $1(2.6)$ & $0(0.0)$ & $0(0.0)$ & \\
\hline Positive & $160(98.8)$ & 46 (97.9) & 38 (97.4) & $24(100.0)$ & $52(100.0)$ & \\
\hline SDHA & & & & & & 0.175 \\
\hline Negative & $2(1.2)$ & $2(4.3)$ & $0(0.0)$ & $0(0.0)$ & $0(0.0)$ & \\
\hline Positive & $160(98.8)$ & 45 (95.7) & $39(100.0)$ & $24(100.0)$ & $52(100.0)$ & \\
\hline SDHB & & & & & & 0.006 \\
\hline Negative & $52(32.1)$ & $20(42.6)$ & $16(41.0)$ & $9(37.5)$ & $7(13.5)$ & \\
\hline Positive & $110(67.9)$ & $27(57.4)$ & $23(59.0)$ & $15(62.5)$ & 45 (86.5) & \\
\hline PHGDH & & & & & & 0.027 \\
\hline Negative & $61(37.7)$ & $24(51.1)$ & $11(28.2)$ & $12(50.0)$ & $14(26.9)$ & \\
\hline Positive & $101(62.3)$ & $23(48.9)$ & 28 (71.8) & $12(50.0)$ & $38(73.1)$ & \\
\hline PSPH & & & & & & 0.926 \\
\hline Negative & $146(90.1)$ & $42(89.4)$ & 36 (92.3) & $22(91.7)$ & 46 (88.5) & \\
\hline Positive & $16(9.9)$ & $5(10.6)$ & $3(7.7)$ & $2(8.3)$ & $6(11.5)$ & \\
\hline PSAT1 & & & & & & $<0.001$ \\
\hline Negative & $140(86.4)$ & $41(87.2)$ & 37 (94.9) & 14 (58.3) & 48 (92.3) & \\
\hline Positive & 22 (13.6) & $6(12.8)$ & $2(5.1)$ & $10(41.7)$ & $4(7.7)$ & \\
\hline
\end{tabular}


Table 3 Expression of metabolism-related proteins in the tumor cell compartment of breast cancer metastasis according to metastatic site (Continued)

\begin{tabular}{|c|c|c|c|c|c|c|}
\hline SHMT1 & & & & & & 0.033 \\
\hline Negative & $127(78.4)$ & $43(91.5)$ & $31(79.5)$ & $18(75.0)$ & 35 (67.3) & \\
\hline Positive & 35 (21.6) & $4(8.5)$ & $8(20.5)$ & $6(25.0)$ & $17(32.7)$ & \\
\hline GLDC & & & & & & 0.547 \\
\hline Negative & $96(59.3)$ & $31(66.0)$ & $24(61.5)$ & $14(58.3)$ & 27 (51.9) & \\
\hline Positive & 66 (40.7) & $16(34.0)$ & 15 (38.5) & $10(41.7)$ & $25(48.1)$ & \\
\hline
\end{tabular}

Bold represents $p<0.05$.

Table 4 Expression of metabolism-related proteins in the stromal compartment of breast cancer metastasis according to metastatic site

\begin{tabular}{|c|c|c|c|c|c|c|}
\hline Parameters & $\begin{array}{l}\text { Total } \\
\mathrm{N}=162(\%)\end{array}$ & $\begin{array}{l}\text { Bone metastasis } \\
n=47(\%)\end{array}$ & $\begin{array}{l}\text { Brain metastasis } \\
\mathrm{n}=39(\%)\end{array}$ & $\begin{array}{l}\text { Liver metastasis } \\
\mathrm{n}=24(\%)\end{array}$ & $\begin{array}{l}\text { Lung metastasis } \\
\mathrm{n}=52(\%)\end{array}$ & p-value \\
\hline Hexokinase II & & & & & & 0.058 \\
\hline Negative & $159(98.1)$ & 44 (93.6) & $39(100.0)$ & $24(100.0)$ & $52(100.0)$ & \\
\hline Positive & $3(1.9)$ & $3(6.4)$ & $0(0.0)$ & $0(0.0)$ & $0(0.0)$ & \\
\hline MCT4 & & & & & & 0.002 \\
\hline Negative & $113(69.8)$ & $26(55.3)$ & $36(92.3)$ & $17(70.8)$ & $34(65.4)$ & \\
\hline Positive & $49(30.2)$ & $21(44.7)$ & $3(7.7)$ & $7(29.2)$ & 18 (34.6) & \\
\hline GLS1 & & & & & & 0.006 \\
\hline Negative & 157 (96.9) & $42(89.4)$ & 39 (100.0) & $24(100.0)$ & $52(100.0)$ & \\
\hline Positive & $5(3.1)$ & 5 (10.6) & $0(0.0)$ & $0(0.0)$ & $0(0.0)$ & \\
\hline $\mathrm{GDH}$ & & & & & & 0.035 \\
\hline Negative & $140(86.4)$ & $36(76.6)$ & 38 (97.4) & $22(91.7)$ & 44 (84.6) & \\
\hline Positive & 22 (13.6) & $11(23.4)$ & $1(2.6)$ & $2(8.3)$ & $8(15.4)$ & \\
\hline ATP synthase & & & & & & 0.084 \\
\hline Negative & 155 (95.7) & $42(89.4)$ & $38(97.4)$ & $24(100.0)$ & $51(98.1)$ & \\
\hline Positive & 7 (4.3) & 5 (10.6) & $1(2.6)$ & $0(0.0)$ & $1(1.9)$ & \\
\hline SDHA & & & & & & 0.004 \\
\hline Negative & 145 (89.5) & $36(76.6)$ & 38 (97.4) & $21(87.5)$ & $50(96.2)$ & \\
\hline Positive & 17 (10.5) & $11(23.4)$ & $1(2.6)$ & $3(12.5)$ & $2(3.8)$ & \\
\hline $\mathrm{PHGDH}$ & & & & & & 0.018 \\
\hline Negative & $158(97.5)$ & $43(91.5)$ & 39 (100.0) & $24(100.0)$ & $52(100.0)$ & \\
\hline Positive & $4(2.5)$ & $4(8.5)$ & $0(0.0)$ & $0(0.0)$ & $0(0.0)$ & \\
\hline PSPH & & & & & & 0.006 \\
\hline Negative & 156 (96.3) & 46 (97.9) & $34(87.2)$ & $24(100.0)$ & $52(100.0)$ & \\
\hline Positive & $6(3.7)$ & $1(2.1)$ & 5 (12.8) & $0(0.0)$ & $0(0.0)$ & \\
\hline PSAT1 & & & & & & $<0.001$ \\
\hline Negative & 154 (95.1) & $46(97.9)$ & $32(82.1)$ & $24(100.0)$ & $52(100.0)$ & \\
\hline Positive & $8(4.9)$ & $1(2.1)$ & 7 (17.9) & $0(0.0)$ & $0(0.0)$ & \\
\hline SHMT1 & & & & & & $<0.001$ \\
\hline Negative & $98(60.5)$ & $16(34.0)$ & $31(79.5)$ & $16(66.7)$ & 35 (67.3) & \\
\hline Positive & $64(39.5)$ & $31(66.0)$ & $8(20.5)$ & 8 (33.3) & $17(32.7)$ & \\
\hline
\end{tabular}




\section{Materials and methods Patient selection}

Patients with invasive primary breast cancer and metastasis to distant organs (lung, bone, brain, and liver) were selected from medical records of the Department of Pathology of Severance Hospital. Only patients with a diagnosis of invasive ductal carcinoma were included. In total, 162 cases were identified, and 49 cases were paired between primary cancer and metastatic cancer. All slides were reviewed, and pathologic diagnoses were approved by two pathologists (JSK and WHJ). Histological grade was assessed using the Nottingham grading system [20]. This study was approved by the Institutional Review Board (IRB) of Severance Hospital. Written informed consent was obtained from the patient for the publication of this report and any accompanying images.

\section{Tissue microarray}

On H\&E-stained slides of the tumors, a representative area was selected, and the corresponding spot was marked on the surface of the paraffin block. Using a biopsy needle, a 3-mm tissue core in the selected area was punched out and placed onto a 65 recipient block. Two tissue cores were extracted to minimize extraction bias. Each tissue core was assigned a unique tissue microarray location number that was linked to a database containing other clinicopathologic data.

\section{Immunohistochemistry (IHC)}

The antibodies used for IHC in this study are shown in Table 1. Formalin-fixed, paraffin-embedded (FFPE) tissue samples were used as follows. Three-micron-thick slices from the FFPE tissue block were deparaffinized and rehydrated in xylene and alcohol solutions and stained using a Ventana Discovery XT automated stainer
(Ventana Medical Systems, Tucson, AZ, USA). Antigen retrieval was performed with CC1 (Cell Conditioning 1) buffer (citrate buffer pH 6.0, Ventana Medical Systems). Appropriate positive and negative controls were used.

\section{Interpretation of immunohistochemical results}

A cut-off value of $1 \%$ or more positively stained nuclei was used to define ER and AR positivity [21]. HER-2 staining was analyzed according to the American Society of Clinical Oncology (ASCO)/College of American Pathologists (CAP) guidelines using the following categories: $0=$ no immunostaining; $1+=$ weak incomplete membranous staining, less than $10 \%$ of tumor cells; $2+=$ complete membranous staining, either uniform or weak in at least $10 \%$ of tumor cells; and $3+=$ uniform intense membranous staining in at least $30 \%$ of tumor cells [22]. HER-2 staining was considered positive when strong $(3+)$ membranous staining was observed whereas it was considered negative when none or weak (0 to $1+$ ) staining was noted.

IHC result interpretation was based on the product of the proportion of stained cells and the immunhistochemical staining intensity. A product between 0 and 1 was regarded as negative, a product between 2 and 4 as low positive, and a product between 5 and 6 as high positive [23]. The proportion of stained cells was scored as 0 for negative, 1 for positive with less than 30\%, and 2 for positive with greater than or equal to $30 \%$. The staining intensity was scored as 0 for negative, 1 for weak, 2 for moderate, and 3 for strong. Ki-67 labeling index (LI) was defined as the percentage of positive cells in tumor cell nuclei.

\section{Tumor phenotype classification}

In this study, breast cancer phenotypes were classified according to IHC results for ER, PR, HER-2, and Ki-67,

Table 5 Metabolic phenotypes of breast cancer metastasis according to metastatic site

\begin{tabular}{|c|c|c|c|c|c|c|}
\hline Parameters & $\begin{array}{l}\text { Total } \\
N=162(\%)\end{array}$ & $\begin{array}{l}\text { Bone metastasis } \\
n=47(\%)\end{array}$ & $\begin{array}{l}\text { Brain metastasis } \\
\mathrm{n}=39(\%)\end{array}$ & $\begin{array}{l}\text { Liver metastasis } \\
\mathrm{n}=24(\%)\end{array}$ & $\begin{array}{l}\text { Lung metastasis } \\
\mathrm{n}=52(\%)\end{array}$ & p-value \\
\hline Glycolysis type & & & & & & $<0.001$ \\
\hline No & $81(50.0)$ & $36(76.6)$ & $9(23.1)$ & $17(70.8)$ & $19(36.5)$ & \\
\hline Yes & $81(50.0)$ & $11(23.4)$ & $30(76.9)$ & $7(29.2)$ & $33(63.5)$ & \\
\hline Glutamine type & & & & & & 0.308 \\
\hline No & $62(38.4)$ & $23(48.9)$ & $14(35.9)$ & $9(37.5)$ & $16(30.8)$ & \\
\hline Yes & $100(61.7)$ & $24(51.1)$ & $25(64.1)$ & $15(62.5)$ & $36(69.2)$ & \\
\hline Mitochondrial type & & & & & & 0.175 \\
\hline No & $2(1.2)$ & $2(4.3)$ & $0(0.0)$ & $0(0.0)$ & $0(0.0)$ & \\
\hline Yes & $160(98.8)$ & 45 (95.7) & $39(100.0)$ & $24(100.0)$ & $52(100.0)$ & \\
\hline Serine/glycine type & & & & & & 0.444 \\
\hline No & $128(79.0)$ & $37(78.7)$ & $34(87.2)$ & $19(79.2)$ & $38(73.1)$ & \\
\hline Yes & $34(21.0)$ & $10(21.3)$ & $5(12.8)$ & $5(20.8)$ & $14(26.9)$ & \\
\hline
\end{tabular}

Bold represents $p<0.05$. 
Table 6 Correlation of expression of metabolism related proteins between primary and metastatic breast cancer according to metastatic site

\begin{tabular}{|c|c|c|c|c|c|c|c|c|c|c|}
\hline \multirow[t]{2}{*}{ Parameters } & \multicolumn{2}{|l|}{ Total } & \multicolumn{2}{|c|}{ Bone metastasis } & \multicolumn{2}{|c|}{ Brain metastasis } & \multicolumn{2}{|c|}{ Liver metastasis } & \multicolumn{2}{|c|}{ Lung metastasis } \\
\hline & $\mathrm{N}=49(\%)$ & $\mathrm{p}$-value & $n=13(\%)$ & p-value & $\mathrm{n}=9(\%)$ & $p$-value & $n=4(\%)$ & $p$-value & $n=23(\%)$ & $\mathrm{p}$-value \\
\hline Glut-1 & & 0.481 & & 0.063 & & 1.000 & & 1.000 & & 0.004 \\
\hline$(+) \rightarrow(+)$ & $10(20.4)$ & & $0(0.0)$ & & $4(44.4)$ & & $0(0.0)$ & & $6(20.4)$ & \\
\hline$(+) \rightarrow(-)$ & $7(14.3)$ & & $5(38.5)$ & & $1(11.1)$ & & $1(25.0)$ & & $0(0.0)$ & \\
\hline$(-) \rightarrow(+)$ & $11(22.4)$ & & $0(0.0)$ & & $2(22.2)$ & & $0(0.0)$ & & $9(39.1)$ & \\
\hline$(-) \rightarrow(-)$ & $21(42.9)$ & & $8(61.5)$ & & $2(22.2)$ & & $3(75.0)$ & & $8(34.8)$ & \\
\hline Hexokinase II & & 0.581 & & 1.000 & & 0.500 & & 1.000 & & 1.000 \\
\hline$(+) \rightarrow(+)$ & $11(22.4)$ & & $1(7.7)$ & & $1(11.1)$ & & $0(0.0)$ & & $9(39.1)$ & \\
\hline$(+) \rightarrow(-)$ & $5(10.2)$ & & $3(23.1)$ & & $0(0.0)$ & & $0(0.0)$ & & $2(8.7)$ & \\
\hline$(-) \rightarrow(+)$ & $8(16.3)$ & & $2(15.4)$ & & $2(22.2)$ & & $1(25.0)$ & & $3(13.0)$ & \\
\hline$(-) \rightarrow(-)$ & $25(51.0)$ & & $7(53.8)$ & & $6(66.7)$ & & $3(75.0)$ & & $9(39.1)$ & \\
\hline CAIX & & 0.688 & & $\mathrm{~N} / \mathrm{A}$ & & 1.000 & & N/A & & 1.000 \\
\hline$(+) \rightarrow(+)$ & $2(4.1)$ & & $0(0.0)$ & & $0(0.0)$ & & $0(0.0)$ & & $2(8.7)$ & \\
\hline$(+) \rightarrow(-)$ & $2(4.1)$ & & $0(0.0)$ & & $1(11.1)$ & & $0(0.0)$ & & $1(4.3)$ & \\
\hline$(-) \rightarrow(+)$ & $4(8.2)$ & & $0(0.0)$ & & $2(22.2)$ & & $0(0.0)$ & & $2(8.7)$ & \\
\hline$(-) \rightarrow(-)$ & $41(83.7)$ & & $13(100.0)$ & & $6(66.7)$ & & $4(100.0)$ & & 18 (78.3) & \\
\hline MCT4 & & 0.002 & & 0.250 & & 0.250 & & 0.500 & & 0.004 \\
\hline$(+) \rightarrow(+)$ & 14 (28.6) & & $3(23.1)$ & & $4(44.4)$ & & $0(0.0)$ & & $7(30.4)$ & \\
\hline$(+) \rightarrow(-)$ & $2(4.1)$ & & $0(0.0)$ & & $0(0.0)$ & & $2(50.0)$ & & $0(0.0)$ & \\
\hline$(-) \rightarrow(+)$ & 15 (30.6) & & $3(23.1)$ & & $3(33.3)$ & & $0(0.0)$ & & $9(39.1)$ & \\
\hline$(-) \rightarrow(-)$ & 18 (36.7) & & $7(53.8)$ & & $2(22.2)$ & & $2(50.0)$ & & $7(30.4)$ & \\
\hline GLS1 & & 1.000 & & 0.063 & & 1.000 & & 1.000 & & 0.289 \\
\hline$(+) \rightarrow(+)$ & 15 (30.6) & & $2(15.4)$ & & $3(33.3)$ & & $2(50.0)$ & & $8(34.8)$ & \\
\hline$(+) \rightarrow(-)$ & $8(16.3)$ & & $0(0.0)$ & & $1(11.1)$ & & $1(25.0)$ & & $6(26.1)$ & \\
\hline$(-) \rightarrow(+)$ & $9(18.4)$ & & $5(38.5)$ & & $2(22.2)$ & & $0(0.0)$ & & $2(8.7)$ & \\
\hline$(-) \rightarrow(-)$ & $17(34.7)$ & & $6(46.2)$ & & $3(33.3)$ & & $1(25.0)$ & & $7(30.4)$ & \\
\hline GDH & & 1.000 & & 1.000 & & 1.000 & & 1.000 & & 1.000 \\
\hline$(+) \rightarrow(+)$ & 44 (89.8) & & 11 (84.6) & & $8(88.9)$ & & $3(75.0)$ & & $22(95.7)$ & \\
\hline$(+) \rightarrow(-)$ & $2(4.1)$ & & $1(7.7)$ & & $1(11.1)$ & & $0(0.0)$ & & $0(0.0)$ & \\
\hline$(-) \rightarrow(+)$ & $3(6.1)$ & & $1(7.7)$ & & $0(0.0)$ & & $1(25.0)$ & & $1(4.3)$ & \\
\hline$(-) \rightarrow(-)$ & $0(0.0)$ & & $0(0.0)$ & & $0(0.0)$ & & $0(0.0)$ & & $0(0.0)$ & \\
\hline ASCT2 & & 0.092 & & 1.000 & & 0.219 & & 1.000 & & 0.375 \\
\hline$(+) \rightarrow(+)$ & $8(16.3)$ & & $0(0.0)$ & & $0(0.0)$ & & $1(25.0)$ & & $7(30.4)$ & \\
\hline$(+) \rightarrow(-)$ & $10(20.4)$ & & $1(7.7)$ & & $5(55.6)$ & & $0(0.0)$ & & $4(17.4)$ & \\
\hline$(-) \rightarrow(+)$ & $3(6.1)$ & & $1(7.7)$ & & $1(11.1)$ & & $0(0.0)$ & & $1(4.3)$ & \\
\hline$(-) \rightarrow(-)$ & $28(57.1)$ & & 11 (84.6) & & $3(33.3)$ & & $3(75.0)$ & & $11(47.8)$ & \\
\hline ATP synthase & & 1.000 & & 1.000 & & N/A & & N/A & & $\mathrm{N} / \mathrm{A}$ \\
\hline$(+) \rightarrow(+)$ & $48(98.0)$ & & 12 (92.3) & & $9(100.0)$ & & $4(100.0)$ & & $23(100.0)$ & \\
\hline$(+) \rightarrow(-)$ & $1(2.0)$ & & $1(7.7)$ & & $0(0.0)$ & & $0(0.0)$ & & $0(0.0)$ & \\
\hline$(-) \rightarrow(+)$ & $0(0.0)$ & & $0(0.0)$ & & $0(0.0)$ & & $0(0.0)$ & & $0(0.0)$ & \\
\hline$(-) \rightarrow(-)$ & $0(0.0)$ & & $0(0.0)$ & & $0(0.0)$ & & $0(0.0)$ & & $0(0.0)$ & \\
\hline
\end{tabular}


Table 6 Correlation of expression of metabolism related proteins between primary and metastatic breast cancer according to metastatic site (Continued)

\begin{tabular}{|c|c|c|c|c|c|c|c|c|c|c|}
\hline $\mathrm{SDHA}$ & & 1.000 & & 1.000 & & 1.000 & & N/A & & N/A \\
\hline$(+) \rightarrow(+)$ & $44(89.8)$ & & $9(69.2)$ & & $8(88.9)$ & & $4(100.0)$ & & $23(100.0)$ & \\
\hline$(+) \rightarrow(-)$ & $2(4.1)$ & & $2(15.4)$ & & $0(0.0)$ & & $0(0.0)$ & & $0(0.0)$ & \\
\hline$(-) \rightarrow(+)$ & $3(6.1)$ & & $2(15.4)$ & & $1(11.1)$ & & $0(0.0)$ & & $0(0.0)$ & \\
\hline$(-) \rightarrow(-)$ & $0(0.0)$ & & $0(0.0)$ & & $0(0.0)$ & & $0(0.0)$ & & $0(0.0)$ & \\
\hline $\mathrm{SDHB}$ & & 0.263 & & 1.000 & & 1.000 & & 1.000 & & 0.063 \\
\hline$(+) \rightarrow(+)$ & $24(49.0)$ & & $4(30.8)$ & & $3(33.3)$ & & $1(25.0)$ & & $16(69.9)$ & \\
\hline$(+) \rightarrow(-)$ & $7(14.3)$ & & $3(23.1)$ & & $3(33.3)$ & & $1(25.0)$ & & $0(0.0)$ & \\
\hline$(-) \rightarrow(+)$ & $13(26.5)$ & & $3(23.1)$ & & $3(33.3)$ & & $2(50.0)$ & & $5(21.7)$ & \\
\hline$(-) \rightarrow(-)$ & $5(10.2)$ & & $3(23.1)$ & & $0(0.0)$ & & $0(0.0)$ & & $2(8.7)$ & \\
\hline PHGDH & & 0.581 & & 1.000 & & 1.000 & & 1.000 & & 0.688 \\
\hline$(+) \rightarrow(+)$ & $22(44.9)$ & & $3(23.1)$ & & $6(66.7)$ & & $1(25.0)$ & & $12(52.2)$ & \\
\hline$(+) \rightarrow(-)$ & $5(10.2)$ & & $2(15.4)$ & & $1(11.1)$ & & $0(0.0)$ & & $2(8.7)$ & \\
\hline$(-) \rightarrow(+)$ & $8(16.3)$ & & $2(15.4)$ & & $1(11.1)$ & & $1(25.0)$ & & $4(17.4)$ & \\
\hline$(-) \rightarrow(-)$ & $14(28.6)$ & & $6(46.2)$ & & $1(11.1)$ & & $2(50.0)$ & & $5(21.7)$ & \\
\hline PSPH & & 1.000 & & N/A & & 1.000 & & 1.000 & & 1.000 \\
\hline$(+) \rightarrow(+)$ & $1(2.0)$ & & $0(0.0)$ & & $0(0.0)$ & & $0(0.0)$ & & $1(4.3)$ & \\
\hline$(+) \rightarrow(-)$ & $3(6.1)$ & & $0(0.0)$ & & $1(11.1)$ & & $0(0.0)$ & & $2(8.7)$ & \\
\hline$(-) \rightarrow(+)$ & $2(4.1)$ & & $0(0.0)$ & & $0(0.0)$ & & $1(25.0)$ & & $1(4.3)$ & \\
\hline$(-) \rightarrow(-)$ & $43(87.8)$ & & $13(100.0)$ & & $8(88.9)$ & & $3(75.0)$ & & 19 (82.6) & \\
\hline PSAT1 & & 0.607 & & 1.000 & & 1.000 & & 1.000 & & 0.219 \\
\hline$(+) \rightarrow(+)$ & $1(2.0)$ & & $0(0.0)$ & & $0(0.0)$ & & $0(0.0)$ & & $1(4.3)$ & \\
\hline$(+) \rightarrow(-)$ & $9(18.4)$ & & $3(23.1)$ & & $0(0.0)$ & & $1(25.0)$ & & $5(21.7)$ & \\
\hline$(-) \rightarrow(+)$ & $6(12.2)$ & & $2(15.4)$ & & $1(11.1)$ & & $2(50.0)$ & & $1(4.3)$ & \\
\hline$(-) \rightarrow(-)$ & $33(67.3)$ & & $8(61.5)$ & & $8(88.9)$ & & $1(25.0)$ & & 16 (69.6) & \\
\hline SHMT1 & & 1.000 & & 1.000 & & 1.000 & & N/A & & 1.000 \\
\hline$(+) \rightarrow(+)$ & $10(20.4)$ & & $0(0.0)$ & & $2(22.2)$ & & $0(0.0)$ & & $8(34.8)$ & \\
\hline$(+) \rightarrow(-)$ & $2(4.1)$ & & $1(7.7)$ & & $0(0.0)$ & & $0(0.0)$ & & $1(4.3)$ & \\
\hline$(-) \rightarrow(+)$ & $1(2.0)$ & & $0(0.0)$ & & $0(0.0)$ & & $0(0.0)$ & & $1(4.3)$ & \\
\hline$(-) \rightarrow(-)$ & $36(73.5)$ & & $12(92.3)$ & & 7 (77.8) & & $4(100.0)$ & & $13(56.5)$ & \\
\hline GLDC & & 0.143 & & 0.375 & & 1.000 & & 1.000 & & 0.508 \\
\hline$(+) \rightarrow(+)$ & $12(24.5)$ & & $0(0.0)$ & & $3(33.3)$ & & $1(25.0)$ & & $8(34.8)$ & \\
\hline$(+) \rightarrow(-)$ & $5(10.2)$ & & $1(7.7)$ & & $1(11.1)$ & & $0(0.0)$ & & $3(13.0)$ & \\
\hline$(-) \rightarrow(+)$ & $12(24.5)$ & & $4(30.8)$ & & $1(11.1)$ & & $1(25.0)$ & & $6(26.1)$ & \\
\hline$(-) \rightarrow(-)$ & $20(40.8)$ & & $8(61.5)$ & & $4(44.4)$ & & $2(50.0)$ & & $6(26.1)$ & \\
\hline
\end{tabular}

*p-value was calculated by McNemar test. Bold represents $p<0.05$.

as well as FISH results for HER-2 as follows [24]: luminal A type: ER or/and PR positive and HER-2 negative and Ki-67 LI <14\%; luminal B type: (HER-2 negative) ER or/and PR positive and HER-2 negative and Ki-67 LI $\geq 14 \%$, (HER-2 positive) ER or/and PR positive and HER2 overexpressed or/and amplified; HER-2 type: ER and PR negative and HER-2 overexpressed or/and amplified; and triple negative breast cancer (TNBC) type: ER, PR, and HER-2 negative.

\section{Classification of tumor metabolic subtype}

In this study, tumor metabolic subtypes were classified according to IHC results for metabolism-related proteins as follows: Glycolysis type: 3 or more positive 

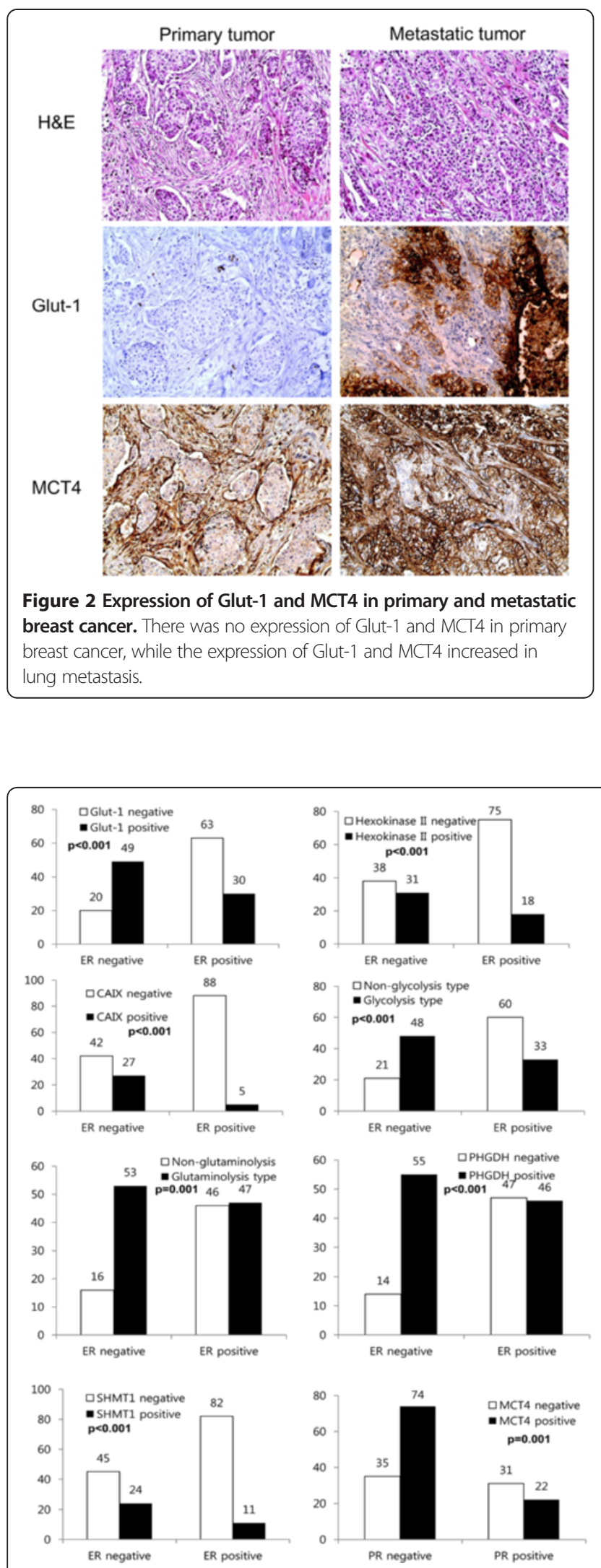

glycolysis-related proteins [Glut-1, hexolinase II, carbonic anhydrase (CA) IX, and monocarboxylate transporter (MCT) 4]; glutaminolysis type: 2 or more positive glutaminolysis-related proteins [glutaminase (GLS) 1, glutamate dehydrogenase (GDH), and amino acid transporter (ASCT) 2]; mitochondrial type: 2 or more positive mitochondrial metabolism proteins [ATP synthase, succinate dehydrogenase (SDH)A, and SDHB]; and serine/glycine type: 3 or more positive serine/glycine metabolism-related proteins [phosphoglycerate dehydrogenase (PHGDH), phosphoserine aminotransferase (PSAT), phosphoserine phosphatase (PSPH), glycine decarboxylase (GLDC), serine hydroxymethyltransferase (SHMT)].

\section{Statistical analysis}

Data were statistically processed using SPSS for Windows, version 12.0 (SPSS Inc., Chicago, IL, USA). Correlation analysis of immunostaining results between primary breast cancer and metastatic breast cancer was calculated by the McNemar test. Students $t$ and Fisher s exact tests were used to examine any differences in continuous and categorical variables, respectively. Corrected $p$-value and the Bonferroni method were used for multiple comparisons. Statistical significance was assumed when $P<0.05$. Kaplan-

Figure 3 Correlation between pathologic factors and expression of metabolism-related proteins. 
Meier survival curves and log-rank statistics were employed to evaluate time to tumor metastasis and time to survival. Multivariate regression analysis was performed using a Cox proportional hazards model.

\section{Results}

Baseline characteristics of patients

In a total of 162 cases, 52 (32.1\%) had lung metastasis, 47 (29.0\%) had bone metastasis, 30 (18.5\%) had brain metastasis, and 24 (14.8\%) had liver metastasis. The proportion of cases with ER positivity and PR positivity was higher among those with bone and liver metastases than in those with metastasis to other sites $(\mathrm{p}<0.001)$, and HER-2 positivity was higher among cases of brain metastasis compared to other sites $(p=0.017)$. Furthermore, luminal A type tumors were more common among patients with bone and liver metastasis, while the proportion of tripe negative breast cancer (TNBC) was higher among cases of brain and lung metastasis $(\mathrm{p}<0.001)($ Table 2$)$.

\section{Expression of metabolism-related proteins in breast} cancer metastasis according to metastatic site (Figure 1)

Analysis of metabolism-related protein expression according to metastatic site in metastatic breast cancer revealed
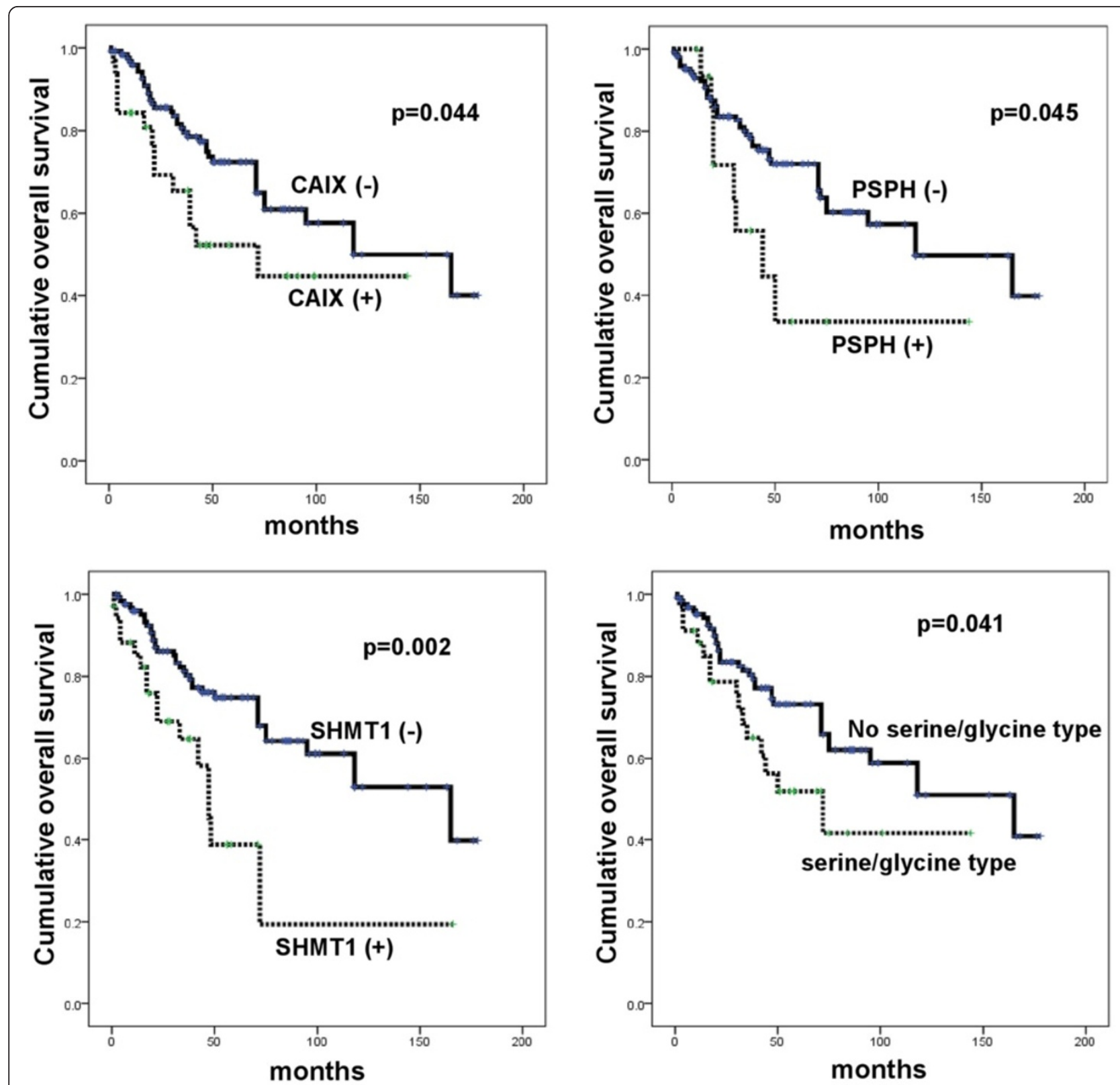

Figure 4 Association between expression level of metabolism-related proteins and patient prognosis in metastatic breast cancer. 
Table 7 Univariate analysis of the association between expression levels of metabolism-related proteins in metastatic breast cancers and overall survival by the log-rank test

\begin{tabular}{|c|c|c|c|c|c|c|c|c|c|c|}
\hline \multirow[t]{2}{*}{ Parameters } & \multicolumn{2}{|l|}{$\begin{array}{l}\text { Total } \\
\mathrm{N}=162(\%)\end{array}$} & \multicolumn{2}{|l|}{$\begin{array}{l}\text { Bone metastasis } \\
n=47(\%)\end{array}$} & \multicolumn{2}{|l|}{$\begin{array}{l}\text { Brain metastasis } \\
\mathrm{n}=39(\%)\end{array}$} & \multicolumn{2}{|l|}{$\begin{array}{l}\text { Liver metastasis } \\
\mathrm{n}=24(\%)\end{array}$} & \multicolumn{2}{|l|}{$\begin{array}{l}\text { Lung metastasis } \\
n=52(\%)\end{array}$} \\
\hline & $\begin{array}{l}\text { Mean survival }(95 \% \mathrm{Cl}) \\
\text { months }\end{array}$ & $\begin{array}{l}P \\
\text {-value }\end{array}$ & $\begin{array}{l}\text { Mean survival }(95 \% \mathrm{Cl}) \\
\text { months }\end{array}$ & $\begin{array}{l}P \\
\text {-value }\end{array}$ & $\begin{array}{l}\text { Mean survival }(95 \% \mathrm{Cl}) \\
\text { months }\end{array}$ & $\begin{array}{l}P \\
\text {-value }\end{array}$ & $\begin{array}{l}\text { Mean survival }(95 \% \mathrm{Cl}) \\
\text { months }\end{array}$ & $\begin{array}{l}P \\
\text {-value }\end{array}$ & $\begin{array}{l}\text { Mean survival }(95 \% \mathrm{Cl}) \\
\text { months }\end{array}$ & $\begin{array}{l}P \\
\text {-value } \\
\end{array}$ \\
\hline Glut-1 & & 0.141 & & 0.020 & & 0.504 & & 0.591 & & 0.833 \\
\hline Negative & $121\left(\begin{array}{ll}103 & 139\end{array}\right)$ & & $102\left(\begin{array}{ll}76 & 129\end{array}\right)$ & & $94\left(\begin{array}{ll}65 & 123\end{array}\right)$ & & $84\left(\begin{array}{ll}64 & 105\end{array}\right)$ & & 131 (97 165) & \\
\hline Positive & $92(72$ 111) & & 56 (41 70) & & 101 (74 127) & & $62(3588)$ & & $120(89$ 150) & \\
\hline Hexokinase II & & 0.727 & & 0.912 & & 0.680 & & 0.418 & & 0.192 \\
\hline Negative & $112(96$ 128) & & $83\left(\begin{array}{ll}61 & 104\end{array}\right)$ & & 103 (76 130) & & $85\left(\begin{array}{ll}65 & 105\end{array}\right)$ & & $146\left(\begin{array}{ll}117 & 175\end{array}\right)$ & \\
\hline Positive & $96\left(\begin{array}{ll}68 & 124\end{array}\right)$ & & $62(42$ 81) & & $54(4265)$ & & $56(2190)$ & & $103(69$ 137) & \\
\hline CAIX & & 0.044 & & $<0.001$ & & 0.527 & & 0.964 & & 0.046 \\
\hline Negative & $115(100130)$ & & $87\left(\begin{array}{ll}67 & 108\end{array}\right)$ & & $115(90 \quad 141)$ & & $81\left(\begin{array}{ll}61 & 101\end{array}\right)$ & & $140(114166)$ & \\
\hline Positive & $80\left(\begin{array}{ll}56 & 103\end{array}\right)$ & & $16\left(\begin{array}{ll}0 & 41\end{array}\right)$ & & $64(4286)$ & & $67\left(\begin{array}{ll}30 & 105\end{array}\right)$ & & $87(49$ 124) & \\
\hline MCT4 & & 0.612 & & 0.787 & & 0.995 & & 0.652 & & 0.456 \\
\hline Negative & $107\left(\begin{array}{ll}87 & 128\end{array}\right)$ & & $92\left(\begin{array}{ll}62 & 122\end{array}\right)$ & & $73\left(\begin{array}{ll}29 & 117\end{array}\right)$ & & $85\left(\begin{array}{ll}63 & 107\end{array}\right)$ & & $120(85$ 154) & \\
\hline Positive & $113\left(\begin{array}{ll}95 & 131\end{array}\right)$ & & $66(5577)$ & & 107 (84 130) & & $61(4082)$ & & $138(107$ 168) & \\
\hline GLS1 & & 0.274 & & 0.690 & & 0.061 & & 0.348 & & 0.896 \\
\hline Negative & $114(96$ 132) & & $85\left(\begin{array}{ll}61 & 108\end{array}\right)$ & & $133(108$ 158) & & $70(5288)$ & & $127(95 \quad 160)$ & \\
\hline Positive & $110(91130)$ & & 61 (43 78) & & $61(4380)$ & & 72 (47 98) & & $133(102$ 164) & \\
\hline $\mathrm{GDH}$ & & 0.919 & & 0.171 & & $\mathrm{n} / \mathrm{a}$ & & $\mathrm{n} / \mathrm{a}$ & & $\mathrm{n} / \mathrm{a}$ \\
\hline Negative & $165(165$ 165) & & $165(165165)$ & & $\mathrm{n} / \mathrm{a}$ & & $\mathrm{n} / \mathrm{a}$ & & $\mathrm{n} / \mathrm{a}$ & \\
\hline Positive & $111\left(\begin{array}{ll}97 & 125\end{array}\right)$ & & $72(58$ 86) & & $\mathrm{n} / \mathrm{a}$ & & $\mathrm{n} / \mathrm{a}$ & & $\mathrm{n} / \mathrm{a}$ & \\
\hline ASCT2 & & 0.686 & & 0.948 & & $\mathrm{n} / \mathrm{a}$ & & 0.761 & & 0.730 \\
\hline Negative & $111(96$ 127) & & $89\left(\begin{array}{ll}64 & 114\end{array}\right)$ & & $\mathrm{n} / \mathrm{a}$ & & $83(62$ 104) & & $135\left(\begin{array}{ll}107 & 162\end{array}\right)$ & \\
\hline Positive & $89(74$ 104) & & $80(47$ 114) & & $\mathrm{n} / \mathrm{a}$ & & $58(4076)$ & & 70 (57 83) & \\
\hline ATP synthase & & 0.965 & & 0.171 & & $\mathrm{n} / \mathrm{a}$ & & $\mathrm{n} / \mathrm{a}$ & & $\mathrm{n} / \mathrm{a}$ \\
\hline Negative & $165(165$ 165) & & $165(165165)$ & & $\mathrm{n} / \mathrm{a}$ & & $\mathrm{n} / \mathrm{a}$ & & $\mathrm{n} / \mathrm{a}$ & \\
\hline Positive & $111\left(\begin{array}{ll}97 & 125\end{array}\right)$ & & $72(58$ 86) & & $\mathrm{n} / \mathrm{a}$ & & $\mathrm{n} / \mathrm{a}$ & & $\mathrm{n} / \mathrm{a}$ & \\
\hline $\mathrm{SDHA}$ & & 0.830 & & 0.132 & & $\mathrm{n} / \mathrm{a}$ & & $\mathrm{n} / \mathrm{a}$ & & $\mathrm{n} / \mathrm{a}$ \\
\hline Negative & $165(165165)$ & & $165(165$ 165) & & $\mathrm{n} / \mathrm{a}$ & & $\mathrm{n} / \mathrm{a}$ & & $\mathrm{n} / \mathrm{a}$ & \\
\hline Positive & $111(96 \quad 125)$ & & $72(5786)$ & & $\mathrm{n} / \mathrm{a}$ & & $\mathrm{n} / \mathrm{a}$ & & $\mathrm{n} / \mathrm{a}$ & \\
\hline
\end{tabular}


Table 7 Univariate analysis of the association between expression levels of metabolism-related proteins in metastatic breast cancers and overall survival by the log-rank test (Continued)

\begin{tabular}{|c|c|c|c|c|c|c|}
\hline SDHB & & 0.460 & 0.630 & 0.372 & 0.870 & 0.649 \\
\hline Negative & $102(80124)$ & $85(52$ 118) & $115(85$ 146) & 49 (45 53) & $82\left(\begin{array}{ll}48 & 115\end{array}\right)$ & \\
\hline Positive & 117 (101 133) & 68 (57 80) & $70(5288)$ & $80\left(\begin{array}{ll}57 & 103\end{array}\right)$ & 135 (112 158) & \\
\hline PHGDH & & 0.590 & 0.494 & 0.878 & 0.048 & 0.939 \\
\hline Negative & $117(96$ 138) & $90\left(\begin{array}{ll}61 & 119\end{array}\right)$ & $84\left(\begin{array}{ll}53 & 115\end{array}\right)$ & $78(6590)$ & $127\left(\begin{array}{ll}80 & 174\end{array}\right)$ & \\
\hline Positive & $108(90 \quad 126)$ & $60(48$ 71) & $107\left(\begin{array}{ll}81 & 133\end{array}\right)$ & 64 (39 89) & $122(89$ 154) & \\
\hline PSPH & & 0.045 & 0.011 & 0.714 & 0.654 & 0.477 \\
\hline Negative & $114(99128)$ & $88\left(\begin{array}{ll}67 & 110\end{array}\right)$ & $108(86$ 131) & $83\left(\begin{array}{ll}63 & 102\end{array}\right)$ & 131 (105 157) & \\
\hline Positive & $68(36$ 100) & 35 (22 49) & $30(1742)$ & $53(2283)$ & $94\left(\begin{array}{ll}41 & 147\end{array}\right)$ & \\
\hline PSAT1 & & 0.542 & $\mathrm{n} / \mathrm{a}$ & $\mathrm{n} / \mathrm{a}$ & 0.927 & 0.074 \\
\hline Negative & 109 (94 123) & $\mathrm{n} / \mathrm{a}$ & $\mathrm{n} / \mathrm{a}$ & $83(59$ 106) & $134(109$ 158) & \\
\hline Positive & 79 (63 95) & $\mathrm{n} / \mathrm{a}$ & $\mathrm{n} / \mathrm{a}$ & 65 (46 83) & 38 (11 65) & \\
\hline SHMT1 & & 0.002 & 0.258 & 0.022 & 0.089 & 0.009 \\
\hline Negative & 119 (104 134) & $84\left(\begin{array}{ll}64 & 105\end{array}\right)$ & $117(95$ 140) & $90(71$ 109) & $147\left(\begin{array}{ll}121 & 172\end{array}\right)$ & \\
\hline Positive & 63 (31 94) & $31(7 \quad 56)$ & $27\left(\begin{array}{ll}19 & 34\end{array}\right)$ & 39 (24 55) & $78(32$ 123) & \\
\hline GLDC & & 0.281 & 0.485 & 0.024 & 0.281 & 0.370 \\
\hline Negative & 115 (98 133) & $80(55$ 104) & $127(105$ 150) & $89\left(\begin{array}{ll}68 & 111\end{array}\right)$ & $135(102$ 168) & \\
\hline Positive & $99(80119)$ & 66 (56 76) & $53(3176)$ & $59(3582)$ & $111(78$ 144) & \\
\hline Glycolysis type & & 0.615 & 0.159 & 0.614 & 0.921 & 0.961 \\
\hline No & $116(97$ 134) & $96\left(\begin{array}{ll}71 & 122\end{array}\right)$ & $91\left(\begin{array}{ll}59 & 123\end{array}\right)$ & $82\left(\begin{array}{ll}61 & 103\end{array}\right)$ & $128(91164)$ & \\
\hline Yes & $99(80118)$ & 63 (51 75) & $102(76$ 128) & 69 (44 94) & $123(95$ 151) & \\
\hline $\begin{array}{l}\text { Glutamine } \\
\text { type }\end{array}$ & & 0.116 & 0.661 & 0.251 & 0.213 & 0.454 \\
\hline No & $121(101$ 142) & $90\left(\begin{array}{ll}59 & 120\end{array}\right)$ & $128(96 \quad 159)$ & 74 (54 93) & 140 (105 175) & \\
\hline Yes & 99 (79 119) & $72\left(\begin{array}{ll}51 & 93\end{array}\right)$ & $68\left(\begin{array}{ll}51 & 84\end{array}\right)$ & 72 (49 95) & $129(101$ 157) & \\
\hline $\begin{array}{l}\text { Mitochondrial } \\
\text { type }\end{array}$ & & 0.830 & 0.132 & $\mathrm{n} / \mathrm{a}$ & $\mathrm{n} / \mathrm{a}$ & $\mathrm{n} / \mathrm{a}$ \\
\hline No & $165(165$ 165) & $165(165165)$ & $\mathrm{n} / \mathrm{a}$ & $\mathrm{n} / \mathrm{a}$ & $\mathrm{n} / \mathrm{a}$ & \\
\hline Yes & $111(96$ 125) & $72(5786)$ & $\mathrm{n} / \mathrm{a}$ & $\mathrm{n} / \mathrm{a}$ & $\mathrm{n} / \mathrm{a}$ & \\
\hline $\begin{array}{l}\text { Serine/glycine } \\
\text { type }\end{array}$ & & 0.041 & 0.886 & 0.467 & 0.034 & 0.019 \\
\hline No & $116(101$ 131) & $83\left(\begin{array}{ll}61 & 105\end{array}\right)$ & 109 (86 133) & $90(72$ 109) & $142(116168)$ & \\
\hline Yes & $79\left(\begin{array}{ll}56 & 103\end{array}\right)$ & $60(4576)$ & $66\left(\begin{array}{ll}30 & 103\end{array}\right)$ & $38(1560)$ & $72(32$ 112) & \\
\hline
\end{tabular}


Table 7 Univariate analysis of the association between expression levels of metabolism-related proteins in metastatic breast cancers and overall survival by the log-rank test (Continued)

\begin{tabular}{|c|c|c|c|c|c|c|}
\hline $\begin{array}{l}\text { Molecular } \\
\text { subtypes }\end{array}$ & & 0.002 & $<0.001$ & 0.081 & N/A & N/A \\
\hline Luminal A & 105 (86 124) & $84\left(\begin{array}{ll}62 & 107\end{array}\right)$ & $55\left(\begin{array}{ll}10 & 100\end{array}\right)$ & N/A & N/A & \\
\hline Luminal B & $140(111$ 170) & 60 (26 93) & $138(112164)$ & N/A & N/A & \\
\hline HER-2 & $134(109$ 158) & 62 (47 77) & 79 (60 97) & N/A & N/A & \\
\hline TNBC & $51(38$ 64) & $3(24)$ & 31 (22 39) & N/A & N/A & \\
\hline
\end{tabular}

Bold represents $\mathrm{p}<0.05$. 
site-specific expression patterns of glycolysis-related proteins (Glut-1, hexokinase II, CAIX, and MCT4), with higher expression levels seen in brain metastasis than in bone or liver metastasis ( $\mathrm{p}<0.001,0.001,0.009$, and $<0.001$, respectively). Similar trends were seen for PHGDH $(\mathrm{p}=0.027)$. The highest expression levels of SDHB and SHMT1 were found in lung metastasis, while the lowest levels were seen in bone metastasis ( $p=0.006$, and 0.033 , respectively) (Table 3 ).

On analysis of metabolism-related protein expression in the stromal compartment of tumors, the expression of MCT4 $(p=0.002)$, GLS1 $(p=0.006)$, GDH $(p=0.035)$, SDHA $(p=0.004)$, PHGDH $(p=0.018)$, PSPH $(p=0.006)$, PSAT1 ( $<0.001)$, and SHMT1 ( $<0.001)$ showed site specificity: higher stromal expression of MCT4, GLS1, GDH, SDHA, PHGDH, and SHMT1 were found in bone metastasis, while PSPH and PSAT1 were higher in brain metastasis (Table 4).

After a review of the metabolic phenotype according to metastatic site, the glycolysis phenotype was most often seen in the brain and lung $(\mathrm{p}<0.001)$ (Table 5).

\section{Correlation of expression of metabolism-related proteins between primary and metastatic breast cancer according to metastatic site}

We analyzed the expression levels of metabolismrelated proteins in primary and metastatic cancers in 49 paired cases. The expression level of MCT4 was statistically different between primary and metastatic cancers $(\mathrm{p}=0.002)$. When considering difference between primary and metastatic cancers according to metastatic sites, Glut-1 ( $\mathrm{p}=0.004)$ and MCT4 $(\mathrm{p}=0.004)$ were expressed in the lung metastasis but not in the primary cancer (Table 6 and Figure 2).

\section{Correlation between pathologic factors and expression of metabolism-related proteins}

On analyzing the association between expression of metabolism-related proteins and pathologic factors, ER negativity was associated with Glut-1 positivity $(\mathrm{p}<0.001)$, hexokinase II positivity $(\mathrm{p}<0.001)$, CAIX positivity $(\mathrm{p}<0.001)$, glycolysis type $(\mathrm{p}<0.001)$, glutaminolysis type $(\mathrm{p}=0.001)$, PHGDH positivity $(\mathrm{p}<0.001)$, and SHMT1 positivity $(\mathrm{p}<0.001)$. PR negativity was associated with MCT4 positivity $(\mathrm{p}=0.001)$ and higher Ki-67 LI was associated with Glut-1 positivity $(\mathrm{p}=0.001)$ and MCT4 positivity $(\mathrm{p}=0.001)$. Glut-1 $(\mathrm{p}<0.001)$, CAIX $(\mathrm{p}<0.001)$, and SHMT1 $(\mathrm{p}<0.001)$ were associated with molecular subtype. If these proteins were expressed, the proportion of TNBC was higher, while luminal A type was higher when these proteins were not expressed. In addition, TNBC was more common in glycolysis type, while luminal A was more common in non-glycolysis type $(\mathrm{p}<0.001)$ (Figure 3$)$.
The association between the expression of metabolism-related proteins and patient prognosis

On analyzing the association of metabolic phenotype and the expression of metabolism-related proteins with patient prognosis with univariate analysis (Figure 4 and Table 7), we found that factors associated with shorter overall survival (OS) were CAIX positivity $(\mathrm{p}=0.044), \mathrm{PSPH}$ positivity $(\mathrm{p}=0.045)$, SHMT1 positivity $(\mathrm{p}=0.002)$, and serine/glycine type $(\mathrm{p}=0.041)$. The factors associated with shorter OS in multivariate analysis were higher Ki-67 LI (hazard ratio: 4.096, 95\% CI: $1.66410 .08, \mathrm{P}=0.002)$ and tumoral SHMT1 positivity (hazard ratio: 2.836, 95\% CI: 1.239 6.495, $\mathrm{P}=0.014$ ) (Table 8).

Univariate analysis was performed to analyze the association between expression of metabolism-related proteins and metabolic phenotype according to metastatic site. The factors associated with shorter OS were Glut-1 positivity $(\mathrm{p}=0.020)$, CAIX positivity $(\mathrm{p}<0.001)$, and PSPH positivity $(\mathrm{p}=0.011)$ in bone metastasis. SHMT1 positivity $(\mathrm{p}=0.022)$ and GLDC positivity $(\mathrm{p}=0.024)$ were associated with shorter OS in brain metastasis, PHGDH positivity $(\mathrm{p}=0.048)$ was associated with shorter OS in liver metastasis, and CAIX positivity $(p=0.046)$ was

Table 8 Multivariate analysis of patient prognosis in metastatic breast cancer

\begin{tabular}{|c|c|c|c|}
\hline \multirow[t]{2}{*}{ Parameters } & \multicolumn{3}{|c|}{ Overall survival } \\
\hline & Hazard ratio & $95 \% \mathrm{Cl}$ & $P$-value \\
\hline ER status & & & 0.067 \\
\hline Negative versus positive & 10.42 & $0.846-128.4$ & \\
\hline PR status & & & 0.091 \\
\hline Negative versus positive & 1.195 & $0.898-4.237$ & \\
\hline HER2 status & & & 0.408 \\
\hline Negative versus positive & 0.436 & $0.075-2.869$ & \\
\hline Ki-67 LI & & & 0.002 \\
\hline$\leq 14$ versus $>14$ & 4.096 & $1.664-10.08$ & \\
\hline Tumor phenotypes & & & 0.147 \\
\hline \multicolumn{4}{|l|}{ Luminal A } \\
\hline Luminal B & 6.697 & $0.387-116.0$ & \\
\hline HER2 & 7.286 & $0.348-152.4$ & \\
\hline TNBC & 0.433 & $0.055-3.387$ & \\
\hline CAIX & & & 0.189 \\
\hline Negative versus positive & 1.690 & $0.773-3.695$ & \\
\hline PSPH & & & 0.117 \\
\hline Negative versus positive & 2.156 & $0.825-5.634$ & \\
\hline SHMT1 & & & 0.014 \\
\hline Negative versus positive & 2.836 & $1.239-6.495$ & \\
\hline Serine/glycine type & & & 0.451 \\
\hline No versus Yes & 0.723 & $0.311-1.679$ & \\
\hline
\end{tabular}

Bold represents $p<0.05$. 
associated with shorter OS in lung metastasis (Figure 5 and Table 7).

\section{Discussion}

In this study, differences in metabolic features of tumors were seen according to metastatic site among cases of metastatic breast cancer. Briefly, brain and lung metastasis showed higher expression levels of glycolysisrelated proteins (Glut-1, hexokinase II, CAIX, and MCT4) than did bone and liver metastasis. Thus, there are several possible reasons for the different metabolic features according to metastatic site. First, the molecular subtypes showed site specificity in metastatic breast cancer, with a high proportion of TNBC in brain and lung metastases and luminal $\mathrm{A}$ in bone and liver metastases. In previous studies, the expression levels of glycolysis-related proteins were higher in TNBC or basal-like type $[25,26]$. Such data are in agreement with the results from this study that the expression of glycolysis-related proteins is higher in brain and lung metastases, which consist of more TNBC cases. Another possible reason is the variety of influences from circulation tumor cells (CTC). Given that CTC, defined as cancer cells in the blood of cancer patients, plays a significant role in the metastatic process, CTC and its metabolites have an influence on metastatic properties. This may result in site specificity according to metastatic site; however, further study is required [27]. One other reason is the different metabolic characteristics of metastatic sites. For example, given that bone tissue creates the hematopoietic cells, the metabolites of bone tissue are expected to differ from those of liver, brain, or lung. This is supported by the fact that immune-responsive tissue and immune-privileged tissues are reported to show different cellular compositions, as well as different metabolic and immunological responses [28].

The stromal expression levels of hexokinase II, MCT4, GLS1, GDH, SDHA, PHGDH, and SHMT1 were higher in
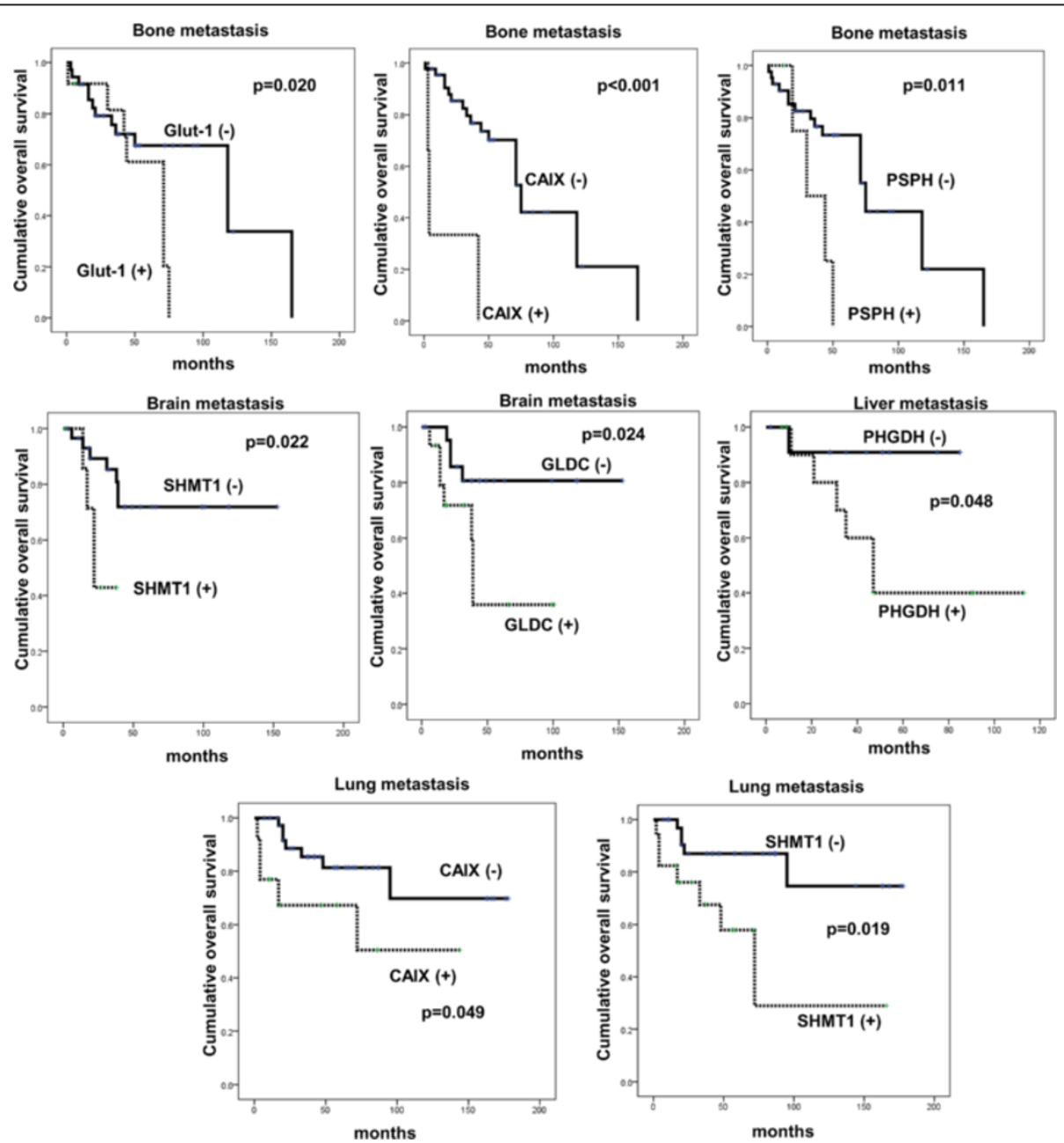

Figure 5 Association between expression level of metabolism-related proteins and patient prognosis in metastatic breast cancer according to the metastatic sites. 
bone metastasis compared to that at other sites. In terms of histologic features, bone metastasis forms highly rich tumor stroma with prominent fibroblasts. The reverseWarburg effect theory, which describes the metabolic interaction between tumor cells and the stroma, supports the expression of metabolism-related proteins in the stroma in bone metastasis. The theory insists that lactate created by glycolysis in the stroma is transferred to tumor cells and metabolized as the substrate by oxidative phosphorylation in tumor cells $[29,30]$. Therefore, the reverse Warburg effect phenotype may be applicable to bone metastasis, in which glycolysisrelated molecules or glycolytic metabolism intermediates are highly expressed. In previous studies, luminal type tumors were more likely to have the reverse Warburg effect phenotype [31]. This may explain why metastatic tumors with the reverse Warburg effect phenotype are more likely to occur in the bone, since luminal type tumors are most commonly found in the bone. However, further validation studies are needed to confirm these findings.

Primary tumors were negative for Glut-1 and MCT4, but some positivity was seen in lung metastasis. Previous studies reported differential expression of most important biomarkers of breast cancer (ER, PR, and HER-2) between primary cancer and metastatic cancer, with $2150 \%$ showing HER-2 loss, about 30\% showing HER-2 gain [8,32], $3.244 \%$ showing ER loss [33-35], 24\% showing PR loss [35], and ER or PR gain not reported. In other words, when primary breast cancer progresses to metastatic cancer, expression of ER/PR can be lost. The loss of ER/PR may be because metastatic cancer exhibits more aggressive features compared to primary tumors; thus, ER/PR, a good prognostic marker, presents as a loss rather than as a gain. In this study, Glut-1 expression was associated with ER negativity and MCT4 expression was associated with PR negativity. In the progression of primary to metastatic cancer, ER/PR is lost and the expression of metabolismrelated proteins like Glut-1 and MCT4 appear. Further validation is required to generalize the findings of this study.

The clinical significance of this study is that inhibition of the metabolic pathway may be a potential treatment target. The expression of metabolism-related proteins, especially glycolysis-related proteins differed according to metastatic site. Previous preclinical studies reported that Glut-1 inhibitor [36,37], CAIX inhibitor [38], and MCT4 inhibitor [39] suppress tumor growth in several tumor types. Thus, these proteins are possible targets for chemotherapy in brain and lung metastasis, which showed higher expression levels of glycolysis-related proteins. However, it should be noted that a compensating response may appear if one or two molecules are inhibited in metabolic pathway targeted therapy [40].

\section{Conclusion}

In conclusion, differences in metabolic features according to metastatic site were seen in metastatic breast cancer, with the glycolysis phenotype found predominantly in the brain and lung since the expression of glycolysis-related protein was higher and the non-glycolysis phenotype in the bone and liver.

\section{Abbreviations}

ER: Estrogen receptor; PR: Progesterone receptor; FFPE: Formalin-fixed, paraffin-embedded; IHC: Immunohistochemistry; ASCO: American Society of Clinical Oncology; CAP: College of American Pathologists; TNBC: Triple negative breast cancer; PHGDH: Phosphoglycerate dehydrogenase; PSAT: Phosphoserine aminotransferase; PSPH: Phosphoserine phosphatase; GLDC: Glycine decarboxylase; SHMT: Serine hydroxymethyltransferase; CAIX: Carbonic anhydrase; MCT: Monocarboxylate transporters; GLS: Glutaminase; GDH: Glutamate dehydrogenase; ASCT: Amino acid transporter; SDH: Succinate dehydrogenase.

\section{Competing interests}

The authors declare that they have no competing interests.

\section{Authors contributions}

HMK participated in the design of the study and performed the statistical analysis and carried out the immunoassays. WHJ participated in its design. JSK conceived the study, and participated in its design and coordination and helped to draft the manuscript. All authors read and approved the final manuscript.

\section{Acknowledgements}

This study was supported by a grant from the National R\&D Program for Cancer Control, Ministry of Health \& Welfare, Republic of Korea (1420080).

Received: 27 August 2014 Accepted: 3 December 2014

Published online: 14 December 2014

\section{References}

1. Weil RJ, Palmieri DC, Bronder JL, Stark AM, Steeg PS: Breast cancer metastasis to the central nervous system. Am J Pathol 2005, 167:913 920.

2. Woodhouse EC, Chuaqui RF, Liotta LA: General mechanisms of metastasis. Cancer 1997, 80:1529 1537.

3. Abali H, Celik I: High incidence of central nervous system involvement in patients with breast cancer treated with epirubicin and docetaxel. Am J Clin Oncol 2002, 25:632 633.

4. Colleoni M, O Neill A, Goldhirsch A, Gelber RD, Bonetti M, Thurlimann B, Price KN, Castiglione-Gertsch M, Coates AS, Lindtner J, Collins J, Senn HJ, Cavalli F, Forbes J, Gudgeon A, Simoncini E, Cortes-Funes H, Veronesi A, Fey M, Rudenstam CM: Identifying breast cancer patients at high risk for bone metastases. J Clin Oncol 2000, 18:3925 3935.

5. Evans AJ, James JJ, Cornford EJ, Chan SY, Burrell HC, Pinder SE, Gutteridge E, Robertson JF, Hornbuckle J, Cheung KL: Brain metastases from breast cancer: identification of a high-risk group. Clin Oncol (R Coll Radiol) 2004, 16:345 349

6. Gaedcke J, Traub F, Milde S, Wilkens L, Stan A, Ostertag H, Christgen M, von Wasielewski R, Kreipe HH: Predominance of the basal type and HER2/neu type in brain metastasis from breast cancer. Mod Pathol 2007, 20:864 870

7. Hicks DG, Short SM, Prescott NL, Tarr SM, Coleman KA, Yoder BJ, Crowe JP, Choueiri TK, Dawson AE, Budd GT, Tubbs RR, Casey G, Weil RJ: Breast cancers with brain metastases are more likely to be estrogen receptor negative, express the basal cytokeratin CK5/6, and overexpress HER2 or EGFR. Am J Surg Pathol 2006, 30:1097 1104.

8. Lorincz T, Toth J, Badalian G, Timar J, Szendroi M: HER-2/neu genotype of breast cancer may change in bone metastasis. Pathol Oncol Res 2006, 12:149 152

9. Nicolson GL: Organ specificity of tumor metastasis: role of preferential adhesion, invasion and growth of malignant cells at specific secondary sites. Cancer Metastasis Rev 1988, 7:143 188. 
10. Paget S: The distribution of secondary growths in cancer of the breast. Lancet 1889, 1:571 572.

11. Hasebe T, Imoto S, Yokose T, Ishii G, I wasaki M, Wada N: Histopathologic factors significantly associated with initial organ-specific metastasis by invasive ductal carcinoma of the breast: a prospective study. Hum Pathol 2008, 39:681 693.

12. Wei B, Wang J, Bourne $P$, Yang $Q$, Hicks D, Bu H, Tang P: Bone metastasis is strongly associated with estrogen receptor-positive/progesterone receptor-negative breast carcinomas. Hum Pathol 2008, 39:1809 1815.

13. Warburg O: On the origin of cancer cells. Science 1956, 123:309 314.

14. Moreno-Sanchez R, Rodriguez-Enriquez S, Marin-Hernandez A, Saavedra E: Energy metabolism in tumor cells. FEBS J 2007, 274:1393 1418.

15. Jain M, Nilsson R, Sharma S, Madhusudhan N, Kitami T, Souza AL, Kafri R, Kirschner MW, Clish CB, Mootha VK: Metabolite profiling identifies a key role for glycine in rapid cancer cell proliferation. Science 2012, 336:1040 1044

16. Bhargava R, Beriwal S, Striebel JM, Dabbs DJ: Breast cancer molecular class ERBB2: preponderance of tumors with apocrine differentiation and expression of basal phenotype markers CK5, CK5/6, and EGFR. Appl Immunohistochem Mol Morphol 2010, 18:113 118.

17. Possemato R, Marks KM, Shaul YD, Pacold ME, Kim D, Birsoy K, Sethumadhavan S, Woo HK, Jang HG, Jha AK, Chen WW, Barrett FG, Stransky N, Tsun ZY, Cowley GS, Barretina J, Kalaany NY, Hsu PP, Ottina K, Chan AM, Yuan B, Garraway LA, Root DE, Mino-Kenudson M, Brachtel EF, Driggers EM, Sabatini DM: Functional genomics reveal that the serine synthesis pathway is essential in breast cancer. Nature 2011, 476:346 350.

18. Zhang WC, Shyh-Chang N, Yang H, Rai A, Umashankar S, Ma S, Soh BS, Sun LL, Tai BC, Nga ME, Bhakoo KK, Jayapal SR, Nichane M, Yu Q, Ahmed DA, Tan C, Sing WP, Tam J, Thirugananam A, Noghabi MS, Pang YH, Ang HS, Mitchell W, Robson P, Kaldis P, Soo RA, Swarup S, Lim EH, Lim B: Glycine decarboxylase activity drives non-small cell lung cancer tumor-initiating cells and tumorigenesis. Cell 2012, 148:259 272.

19. Dang CV, Hamaker M, Sun P, Le A, Gao P: Therapeutic targeting of cancer cell metabolism. J Mol Med (Berl) 2011, 89:205 212.

20. Elston CW, Ellis IO: Pathological prognostic factors in breast cancer. I. The value of histological grade in breast cancer: experience from a large study with long-term follow-up. Histopathology 1991, 19:403 410.

21. Hammond ME, Hayes DF, Dowsett M, Allred DC, Hagerty KL, Badve S, Fitzgibbons PL, Francis G, Goldstein NS, Hayes M, Hicks DG, Lester S, Love R, Mangu PB, McShane L, Miller K, Osborne CK, Paik S, Perlmutter J, Rhodes A, Sasano H, Schwartz JN, Sweep FC, Taube S, Torlakovic EE, Valenstein P, Viale $G$, Visscher D, Wheeler T, Williams RB, et al: American Society of Clinical Oncology/College Of American Pathologists guideline recommendations for immunohistochemical testing of estrogen and progesterone receptors in breast cancer. J Clin Oncol 2010, 28:2784 2795.

22. Wolff AC, Hammond ME, Schwartz JN, Hagerty KL, Allred DC, Cote RJ, Dowsett M, Fitzgibbons PL, Hanna WM, Langer A, MCShane LM, Paik S, Pegram MD, Perez EA, Press MF, Rhodes A, Sturgeon C, Taube SE, Tubbs R, Vance GH, van de Vijver M, Wheeler TM, Hayes DF: American Society of Clinical Oncology/College of American Pathologists guideline recommendations for human epidermal growth factor receptor 2 testing in breast cancer. J Clin Oncol 2007, 25:118 145.

23. Won KY, Kim GY, Kim YW, Song JY, Lim SJ: Clinicopathologic correlation of beclin-1 and bcl-2 expression in human breast cancer. Hum Pathol 2010, 41:107 112.

24. Goldhirsch A, Wood WC, Coates AS, Gelber RD, Thurlimann B, Senn HJ: Strategies for subtypes dealing with the diversity of breast cancer: highlights of the St. Gallen international expert consensus on the primary therapy of early breast cancer 2011. Ann Oncol 2011, 22:1736 1747.

25. Pinheiro C, Sousa B, Albergaria A, Paredes J, Dufloth R, Vieira D, Schmitt F, Baltazar F: GLUT1 and CAIX expression profiles in breast cancer correlate with adverse prognostic factors and MCT1 overexpression. Histol Histopathol 2011, 26:1279 1286.

26. Choi J, Jung WH, Koo JS: Metabolism-related proteins are differentially expressed according to the molecular subtype of invasive breast cancer defined by surrogate immunohistochemistry. Pathobiology 2013, 80:41 52.

27. Bidard FC, Fehm T, Ignatiadis M, Smerage JB, Alix-Panabieres $C$, Janni W Messina C, Paoletti C, Muller V, Hayes DF, Piccart M, Pierga JY: Clinical application of circulating tumor cells in breast cancer: overview of the current interventional trials. Cancer Metastasis Rev 2013, 32:179 188.

28. Khatami M: Unresolved inflammation: immune tsunami or erosion of integrity in immune-privileged and immune-responsive tissues and acute and chronic inflammatory diseases or cancer. Expert Opin Biol Ther 2011, 11:1419 1432.

29. Pavlides S, Tsirigos A, Vera I, Flomenberg N, Frank PG, Casimiro MC, Wang C, Fortina P, Addya S, Pestell RG, Martinez-Outschoorn UE, Sotgia F, Lisanti MP: Loss of stromal caveolin-1 leads to oxidative stress, mimics hypoxia and drives inflammation in the tumor microenvironment, conferring the reverse Warburg effect : a transcriptional informatics analysis with validation. Cell Cycle 2010, 9:2201 2219.

30. Pavlides S, Whitaker-Menezes D, Castello-Cros R, Flomenberg N, Witkiewicz AK, Frank PG, Casimiro MC, Wang C, Fortina P, Addya S, Pestell RG, MartinezOutschoorn UE, Sotgia F, Lisanti MP: The reverse Warburg effect: aerobic glycolysis in cancer associated fibroblasts and the tumor stroma. Cell Cycle 2009, 8:3984 4001.

31. Choi J, Kim Do H, Jung WH, Koo JS: Metabolic interaction between cancer cells and stromal cells according to breast cancer molecular subtype. Breast Cancer Res 2013, 15:R78.

32. Zidan J, Dashkovsky I, Stayerman C, Basher W, Cozacov C, Hadary A: Comparison of HER-2 overexpression in primary breast cancer and metastatic sites and its effect on biological targeting therapy of metastatic disease. Br J Cancer 2005, 93:552 556.

33. Brunn Rasmussen B, Kamby C: Immunohistochemical detection of estrogen receptors in paraffin sections from primary and metastatic breast cancer. Pathol Res Pract 1989, 185:856 859.

34. Gomez-Fernandez C, Daneshbod Y, Nassiri M, Milikowski C, Alvarez C, Nadji M: Immunohistochemically determined estrogen receptor phenotype remains stable in recurrent and metastatic breast cancer. Am J Clin Pathol 2008, 130:879 882.

35. Kuukasjarvi T, Kononen J, Helin H, Holli K, Isola J: Loss of estrogen receptor in recurrent breast cancer is associated with poor response to endocrine therapy. J Clin Oncol 1996, 14:2584 2589.

36. Mohanti BK, Rath GK, Anantha N, Kannan V, Das BS, Chandramouli BA, Banerjee AK, Das S, Jena A, Ravichandran R, Sahi UP, Kumar R, Kapoor N, Kalia VK, Dwarakanath BS, Jain V: Improving cancer radiotherapy with 2-deoxy-D-glucose: phase I/II clinical trials on human cerebral gliomas. Int J Radiat Oncol Biol Phys 1996, 35:103 111.

37. Aft RL, Zhang FW, Gius D: Evaluation of 2-deoxy-D-glucose as a chemotherapeutic agent: mechanism of cell death. Br J Cancer 2002, 87:805 812.

38. Vullo D, Franchi M, Gallori E, Pastorek J, Scozzafava A, Pastorekova S, Supuran CT: Carbonic anhydrase inhibitors: inhibition of the tumorassociated isozyme IX with aromatic and heterocyclic sulfonamides. Bioorg Med Chem Lett 2003, 13:1005 1009.

39. Gallagher SM, Castorino JJ, Wang D, Philp NJ: Monocarboxylate transporter 4 regulates maturation and trafficking of CD147 to the plasma membrane in the metastatic breast cancer cell line MDA-MB-231. Cancer Res 2007, 67:4182 4189.

40. Khatami M: Unresolved inflammation and cancer: loss of natural immune surveillance as the correct target for therapy! seeing the Elephant in the light of logic. Cell Biochem Biophys 2012, 62:501509.

doi:10.1186/s12967-014-0354-3

Cite this article as: Kim et al.: Site-specific metabolic phenotypes in metastatic breast cancer. Journal of Translational Medicine 2014 12:354.

\section{Submit your next manuscript to BioMed Central and take full advantage of:}

$\otimes$ Convenient online submission

$\otimes$ Thorough peer review

\No space constraints or color $\nabla$ gure charges

$\otimes$ Immediate publication on acceptance

\Inclusion in PubMed, CAS, Scopus and Google Scholar

$\otimes$ Research which is freely available for redistribution

Submit your manuscript at www.biomedcentral.com/submit

C) BioMed Central 